\title{
IDENTIFICATION OF MIMO DYNAMIC SYSTEM USING INVERSE MIMO NEURAL NARX MODEL
}

\author{
Ho Pham Huy Anh ${ }^{(1)}$, Nguyen Thanh Nam ${ }^{(2)}$ \\ (1) Ho Chi Minh City University of Technology, VNU-HCM \\ (2) DCSELAB, University of Technology, VNU-HCM \\ (Manuscript Received on April 5 ${ }^{\text {th }}$, 2012, Manuscript Revised May 15 ${ }^{\text {th }}$, 2013)
}

ABSTRACT: This paper investigates the application of proposed neural MIMO NARX model to a nonlinear 2-axes pneumatic artificial muscle (PAM) robot arm as to improve its performance in modeling and identification. The contact force variations and nonlinear coupling effects of both joints of the 2-axes PAM robot arm are modeled thoroughly through the novel dynamic inverse neural MIMO $N A R X$ model exploiting experimental input-output training data. For the first time, the dynamic neural inverse MIMO NARX Model of the 2-axes PAM robot arm has been investigated. The results show that this proposed dynamic intelligent model trained by Back Propagation learning algorithm yields both of good performance and accuracy. The novel dynamic neural MIMO NARX model proves efficient for modeling and identification not only the 2-axes PAM robot arm but also other nonlinear dynamic systems.

Keywords: dynamic modeling, pneumatic artificial muscle (PAM), 2-axes PAM robot arm, inverse identification, neural MIMO NARX model, back propagation (BP) algorithm

\section{INTRODUCTION}

Rehabilitation robots up to now begin to be applied for treatment of patients suffering from trauma or stroke. Since the number of patients is large and the treatment is time consuming, it is a big advantage if rehabilitation robots can assist in performing treatment. Noritsugu et al. [1] designed an arm-like robot for treating patients with trauma, and developed four modes of linear motion with impedance control to control the force during the movement. Krebs et al. [2] designed a planar robot with impedance control for guiding patients to make movements along the specified trajectories. Ju et al. [3] added different constant external loads, by a robot in torque control mode.

Pneumatic Artificial Muscle (PAM) actuators are now used in the various fields of medical robots. The modern robotics toward applications requires greater friendliness between robot actuator and human operator. PAM actuator has achieved increasing belief to the ability of providing advantages such as high power/weight ratio, full of hygiene, easiness in preservation and especially the capacity of human compliance which is the most important requirement in medical and human welfare field. Therefore PAM has been regarded during 
the recent decades as an interesting alternative

to hydraulic and electric actuators. Consequently, PAM-based applications have been published increasingly. Caldwell et al. (2003) in [4] have developed and controlled of a PAM-based Soft-Actuated Exoskeleton for use in physiotherapy and training. Kobayashi et al. (2003) in [5] have applied PAM as to develop a Muscle suit for Upper Body. Noritsugu et al. (2005) in [6] have used PAM for developing an Active Support Splint among them.

Unfortunately, up to now principal difficulty inherent in PAM actuators is the problem of modeling and controlling them efficiently and precisely. This is because they are highly nonlinear and time varying. Since the rubber tube and plastic sheath are continually in contact with each other and the PAM shape is continually changing, the PAM temperature varies with use, changing the properties of the actuator over time. Approaches to PAM modeling and control have included PID control, adaptive control (Lilly, 2003)[7], nonlinear optimal predictive control [8], variable structure control [9], and various soft computing approaches including intelligent model + phase plane switching control (Ahn et al., 2006)[10], neuro-fuzzy model and genetic control in (Carbonell et al., 2001)[11], (Lilly and Chang, 2003)[12] and so on.

Among such advanced modeling and control schemes, as to guarantee a good tracking performance, robust adaptive control approaches combining conventional methods with new learning techniques are required (Lin and Lee, 1991)[13]. Thanks to their universal approximation capabilities, neural networks provide the implementation tool for modeling the complex input-output relations of the multiple $n$ DOF PAM manipulator which is able to solve dynamic problems like variablecoupling complexity and state-dependency. During the last decade several neural network models and learning schemes have been applied to offline learning of manipulator dynamics (Karakasoglu et al., 1993)[14], (Katic et al., 1995)[15], (Lewis et al., 1999)[16], (Boerlage et al., 2003)[17]. In (Pham et al., 2005)[18], authors applied neurofuzzy modeling and control of robot manipulators for trajectory tracking. Ahn and Anh in [19] have optimized successfully a pseudo-linear ARX model of the PAM manipulator using genetic algorithm. These authors in (Anh et al., 2007)[20] have identified the highly nonlinear 2-axes PAM manipulator based on recurrent neural networks. Nevertheless, the drawback of all these results is considered the $n$-DOF manipulator as $n$ independent decoupling joints. Consequently, all intrinsic coupling features of the $n$-DOF manipulator have not represented in its NN model respectively.

To overcome this disadvantage, in this paper, a new approach of neural networks, proposed dynamic inverse neural MIMO NARX model, firstly utilized in simultaneous modeling and identification of the nonlinear 2- 
axes PAM robot arm system. The experiment results have demonstrated the feasibility and good performance of the proposed intelligent inverse model which overcomes successfully external and internal disturbances such as contact force variations and highly nonlinear coupling effects of both joints of the 2-axes PAM robot arm.

The outline of this paper composes of the section 1 for introducing related works in PAM robot arm modeling and identification. The section 2 presents identification procedure of an inverse neural MIMO NARX model using back propagation learning algorithm. The section 3 proves and analyses experimental studies and results considering the contact force variations and highly nonlinear coupling effects of both joints of the nonlinear dynamic system. Finally, the conclusion belongs to the section 4 .

2. IDENTIFICATION USING DYNAMIC INVERSE NEURAL MIMO NARX MODEL

\subsection{Dynamic Neural MIMO NARX Model}

Inverse Neural MIMO NARX model used in this paper is a combination between the Multi-Layer Perceptron Neural Networks (MLPNN) structure and the ARX model. Due to this combination, Inverse MIMO NARX model possesses both of powerful universal approximating feature from MLPNN structure and strong predictive feature from nonlinear ARX model.

A fully connected 3-layer feed-forward MLP-network with $n$ inputs, $q$ hidden units (also called "nodes" or "neurons"), and $m$ outputs units is shown in Fig. 1.

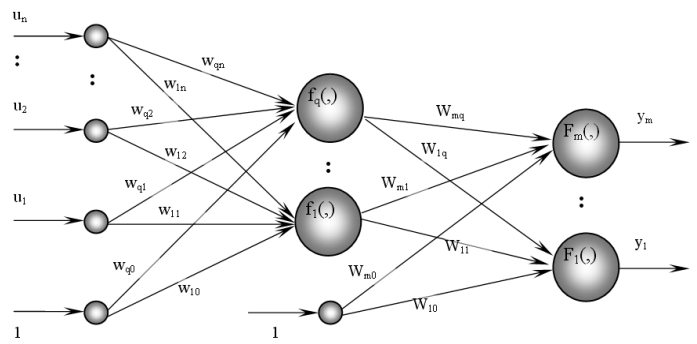

Figure 1. Structure of feed-forward MLPNN

In Fig. $1, w_{10}, . ., w_{q 0}$ and $W_{10}, . ., W_{m 0}$ are weighting values of Bias neurons of Input Layer and Hidden Layer respectively.

Consider an ARX model with noisy input, which can be described as $A\left(q^{-1}\right) y(t)=B\left(q^{-1}\right) u(t-T)+C\left(q^{-1}\right) e(t)$

(1)

with $\quad A\left(q^{-1}\right)=1+a_{1} q^{-1}+a_{2} q^{-2}$

$B\left(q^{-1}\right)=b_{1}+b_{2} q^{-1}$

$C\left(q^{-1}\right)=c_{1}+c_{2} q^{-1}+c_{3} q^{-2}$

where $e(t)$ is the white noise sequence with zero mean and unit variance; $u(t)$ and $y(t)$ are input and output of system respectively; $q$ is the shift operator and $T$ is the time delay.

From equation (1), not consider noise component $e(t)$, we have the general form of the discrete ARX model in domain $z$ (with the time delay $T=n_{k}=1$ )

$\frac{y\left(z^{-1}\right)}{u\left(z^{-1}\right)}=\frac{b_{1} z^{-1}+b_{2} z^{-2}+\ldots+b_{n_{b}} z^{-n_{b}}}{1+a_{1} z^{-1}+a_{2} z^{-2}+\ldots+a_{n_{a}} z^{-n_{a}}}$

in which $n_{a}$ and $n_{b}$ are the order of output $y\left(z^{-1}\right)$ and input $u\left(z^{-1}\right)$ respectively. 
This paper investigates the potentiality of various simple MIMO NARX models in order to exploit them in modeling, identification and control as well. Thus, by embedding a 3-layer MLPNN (with number of neurons of hidden layer $=5)$ in a $2^{\text {nd }}$ order ARX model with its characteristic equation derived from (2) as follows:

$$
\begin{aligned}
& y_{1}(k)=b_{11} u_{1}(k)+b_{12} u_{2}(k)-a_{11} y_{1}(k-1)-a_{12} y_{2}(k-1) \\
& y_{2}(k)=b_{21} u_{1}(k)+b_{22} u_{2}(k)-a_{21} y_{1}(k-1)-a_{22} y_{2}(k-1)
\end{aligned}
$$

We will design the proposed inverse MIMO Neural NARX11 model $\left(n_{a}=1, n_{b}=1\right.$, $n_{k}=1$ ) with 6 inputs (including $u_{11}(t)$ and $u_{12}(t)$ identical to input value $u_{1}(t), u_{21}(t)$ and $u_{22}(t)$ identical to input value $u_{2}(t)$, and recurrent delayed values $\left.y_{1}(t-1), y_{2}(t-1)\right), 2$ output values $\left(y_{\text {lhat }}(t), y_{2 h a t}(t)\right)$. Its structure is shown in Fig. 2.

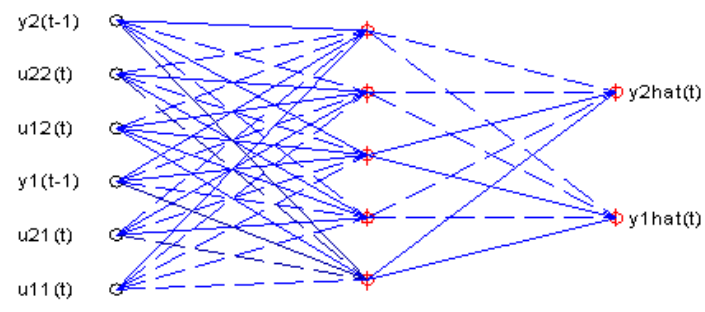

Figure 2. Structure of MIMO Neural NARX11 model

By this way, the parameters $a_{11}, a_{12}, b_{11}$, $b_{12}$ of linear ARX model now become nonlinear and will be determined from the weighting values $W_{i j}$ and $w_{j l}$ of the nonlinear MIMO Neural NARX model. This feature makes MIMO Neural NARX model very powerful in modeling, identification and in model-based advanced control as well.

The class of MLPNN-networks considered in this paper is furthermore confined to those having only one hidden layer and using sigmoid activation functions. From Fig.1, predictive output value $\hat{y}(t)$ is calculated as follows:

$$
\begin{aligned}
& \hat{y}_{i}(w, W)=F_{i}\left(\sum_{j=1}^{q} W_{i j} O_{j}(w)+W_{i 0}\right)= \\
& F_{i}\left(\sum_{j=1}^{q} W_{i j} f_{j}\left(\sum_{l=1}^{n} w_{j l} z_{l}+w_{j 0}\right)+W_{i 0}\right)
\end{aligned}
$$

The weights are the adjustable parameters of the network, and they are determined from a set of examples through the process called training. The examples, or the training data as they are usually called, are a set of inputs, $u(t)$, and corresponding desired outputs, $y(t)$.

Specify the training set by:

$$
Z^{N}=\{[u(t), y(t)] \mid t=1, \ldots, N\}
$$

The objective of training is then to determine a mapping from the set of training data to the set of possible weights: $Z^{N} \rightarrow \hat{\theta}$ so that the network will produce predictions $\hat{y}(t)$, which in some sense are "closest" to the true joint angle outputs $y(t)$ of PAM robot arm.

The prediction error approach, which is the strategy applied here, is based on the introduction of a measure of closeness in terms of a mean sum of square error (MSSE) criterion:

$$
\begin{aligned}
& E_{N}\left(\theta, Z^{N}\right)= \\
& \frac{1}{2 N} \sum_{t=1}^{N}[y(t)-\hat{y}(t \mid \theta)]^{T}[y(t)-\hat{y}(t \mid \theta)]^{(6)}
\end{aligned}
$$

\section{Trang 16}


Based on the conventional error BackPropagation (BP) training algorithms, the weighting value is calculated as follows:

$W(k+1)=W(k)-\lambda \frac{\partial E(W(k))}{\partial W(k)}$

with $k$ is $k^{\text {th }}$ iterative step of calculation and $\lambda$. is learning rate which is often chosen as a small constant value.

Concretely, the weights $W_{i j}$ and $w_{j l}$ of neural NARX structure are then updated as:

$W_{i j}(k+1)=W_{i j}(k)+\Delta W_{i j}(k+1)$

$\Delta W_{i j}(k+1)=\lambda \cdot \delta_{i} \cdot O_{j}$

$\delta_{i}=\hat{y}_{i}\left(1-\hat{y}_{i}\right)\left(y_{i}-\hat{y}_{i}\right)$

with $\delta_{i}$ is search direction value of $i^{t h}$ neuron of output layer $(i=[1 \rightarrow m]) ; O_{j}$ is the output value of $j^{\text {th }}$ neuron of hidden layer $(j=[1 \rightarrow q]) ; y_{i}$ and $\hat{y}_{i}$ are truly real output and predicted output of $i^{\text {th }}$ neuron of output layer $(i=[1 \rightarrow m])$, and

$w_{j l}(k+1)=w_{j l}(k)+\Delta w_{j l}(k+1)$

$\Delta w_{j l}(k+1)=\lambda \cdot \delta_{j} \cdot u_{l}$

$\delta_{j}=O_{j}\left(1-O_{j}\right) \sum_{i=1}^{m} \delta_{i} W_{i j}$

in which $\delta_{j}$ is search direction value of $j^{t h}$ neuron of hidden layer $(j=[1 \rightarrow q]) ; O_{j}$ is the output value of $j^{\text {th }}$ neuron of hidden layer $(j=[l \rightarrow q]) ; u_{l}$ is input of $l^{\text {th }}$ neuron of input layer $(l=[1 \rightarrow n])$.

These results of equations (8) and (9) are demonstrated as follow in case of sigmoid being activate function of hidden and output layer. Consider in case of output layer:
Error to be minimized:

$$
E=\frac{1}{2} \sum_{i=1}^{m}\left(\hat{y}_{i}-y_{i}\right)^{2}
$$

Using chain rule method, we have:

$$
\frac{\partial E}{\partial W_{i j}}=\frac{\partial E}{\partial \hat{y}_{i}} \frac{\partial \hat{y}_{i}}{\partial S_{i}} \frac{\partial S_{i}}{\partial W_{i j}}
$$

From equation (10), the following equation is derived.

$$
\frac{\partial E}{\partial \hat{y}_{i}}=\left(\hat{y}_{i}-y_{i}\right)
$$

with $S_{i}=\sum_{j=1}^{q} W_{i j} \cdot O_{j}+$ bias $_{i}$ as sum calculation at $i^{\text {th }}$ node of output layer and $\hat{y}_{i}=\frac{1}{1+e^{-S_{i}}}$, it gives

$$
\frac{\partial \hat{y}_{i}}{\partial S_{i}}=\frac{e^{-S_{i}}+1-1}{\left(1+e^{-S_{i}}\right)^{2}}=\frac{1}{\left(1+e^{-S_{i}}\right)}\left(1+\frac{-1}{1+e^{-S_{i}}}\right)=
$$$$
\hat{y}_{i}\left(1-\hat{y}_{i}\right)
$$

$$
\frac{\partial S_{i}}{\partial W_{i j}}=O_{j}
$$

Replace (12), (13), (14) to (11) and then put all to (7), the following equation is derived.

$W_{i j}(k+1)=W_{i j}(k)+\Delta W_{i j}(k+1)$

$\Delta W_{i j}(k+1)=\lambda \cdot \delta_{i} \cdot O_{j}$

$\delta_{i}=\hat{y}_{i}\left(1-\hat{y}_{i}\right)\left(y_{i}-\hat{y}_{i}\right)$

Equation (8) has been demonstrated.

The same way for updating the weights of hidden layer, using the chain rule method, we have: 


$$
\frac{\partial E}{\partial w_{j l}}=\frac{\partial E}{\partial O_{j}} \frac{\partial O_{j}}{\partial S_{j}} \frac{\partial S_{j}}{\partial w_{j l}}
$$

Then

$$
\begin{aligned}
& \frac{\partial E}{\partial O_{j}}=\sum_{i=1}^{m}\left[\frac{\partial E}{\partial S_{i}} \frac{\partial S_{i}}{\partial O_{j}}\right]= \\
& \sum_{i=1}^{m}\left[\frac{\partial E}{\partial S_{i}} \frac{\partial}{\partial O_{j}}\left\{\sum_{j=1}^{q} W_{i j} O_{j}+\text { bias }_{i}\right\}\right]= \\
& \sum_{i=1}^{m}\left[\frac{\partial E}{\partial S_{i}} W_{i j}\right]=\sum_{i=1}^{m}\left[-\delta_{i} W_{i j}\right]
\end{aligned}
$$

$$
\text { with } S_{j}=\sum_{l=1}^{n} w_{j l} \cdot u_{l}+\text { bias }_{j} \text { as sum }
$$
calculation at $j^{\text {th }}$ node of hidden layer and

$$
\begin{aligned}
& O_{j}=\frac{1}{1+e^{-S_{j}}} \text {, it gives } \\
& \frac{\partial O_{j}}{\partial S_{j}}=\frac{e^{-S_{j}}+1-1}{\left(1+e^{-S_{j}}\right)^{2}}=\frac{1}{\left(1+e^{-S_{j}}\right)}\left(1+\frac{-1}{1+e^{-S_{j}}}\right)= \\
& O_{j}\left(1-O_{j}\right) \\
& \quad \frac{\partial S_{j}}{\partial w_{j l}}=u_{l}
\end{aligned}
$$

Replace (17), (18), (19) to (16) and then put all to (7), the following equation is derived.

$$
\begin{aligned}
& w_{j l}(k+1)=w_{j l}(k)+\Delta w_{j l}(k+1) \\
& \Delta w_{j l}(k+1)=\lambda \cdot \delta_{j} \cdot u_{l} \\
& \delta_{j}=O_{j}\left(1-O_{j}\right) \sum_{i=1}^{m} \delta_{i} W_{i j}
\end{aligned}
$$

Equation (9) has been demonstrated.

\subsection{Experiment Set Up}

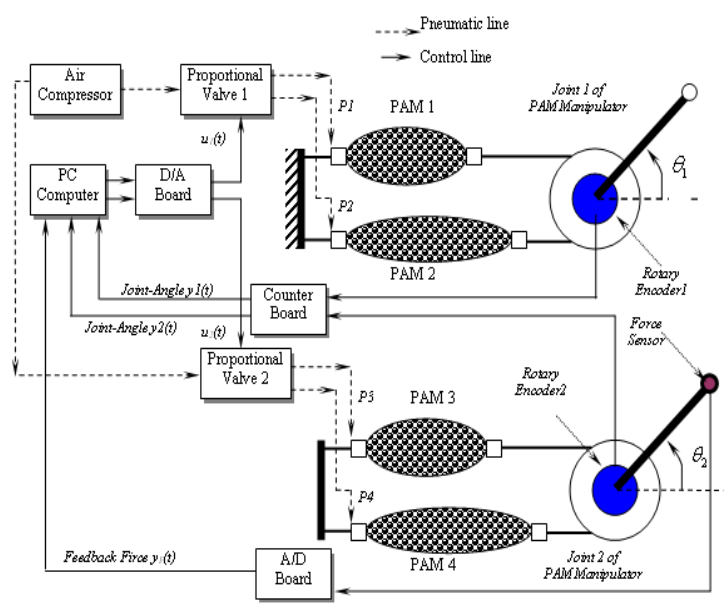

Figure 3. Block diagram for working principle of the 2-axes PAM robot arm.

A general configuration of the investigated 2-axes PAM robot arm shown through the schematic diagram of the 2-axes PAM robot arm and the photograph of the experimental apparatus are shown in Fig.3 and Fig.4, respectively. Both of joints of the 2-axes PAM robot arm are modeled and identified simultaneously through proposed neural MIMO NARX model.

The hardware includes an IBM compatible PC (Pentium 1.7 GHz) which sends the voltage signals $u_{1}(t)$ and $u_{2}(t)$ to control the two proportional valves (FESTO, MPYE-5-1/8HF710B), through a D/A board (ADVANTECH, PCI 1720 card) which changes digital signals from PC to analog voltage $u_{1}(t)$ and $u_{2}(t)$ respectively. The rotating torque is generated by the pneumatic pressure difference supplied from air-compressor between the antagonistic artificial muscles. Consequently, the both of

\section{Trang 18}


joints of the 2-axes PAM robot arm will be rotated to follow the desired joint angle references $\left(Y_{R E F I}(k)\right.$ and $\left.Y_{R E F 2}(k)\right)$ respectively. The joint angles, $\theta_{1}[\mathrm{deg}]$ and $\theta_{2}[\mathrm{deg}]$, are detected by two rotary encoders (METRONIX, H40-8-3600ZO) and fed back to the computer through a 32-bit counter board (COMPUTING MEASUREMENT, PCI QUAD-4 card) which changes digital pulse signals to joint angle values $y_{1}(t)$ and $y_{2}(t)$. Simultaneously, through an A/D board (ADVANTECH, PCI 1710 card) which will send to PC the external force value which is detected by a force sensor CBFS-10. The pneumatic line is conducted under the pressure of 5[bar] and the software control algorithm of the closed-loop system is coded in C-mex program code run in Real-Time Windows Target of MATLAB-SIMULINK environment. Table 1 presents the configuration of the hardware set-up installed from Fig.3, and Fig.4.

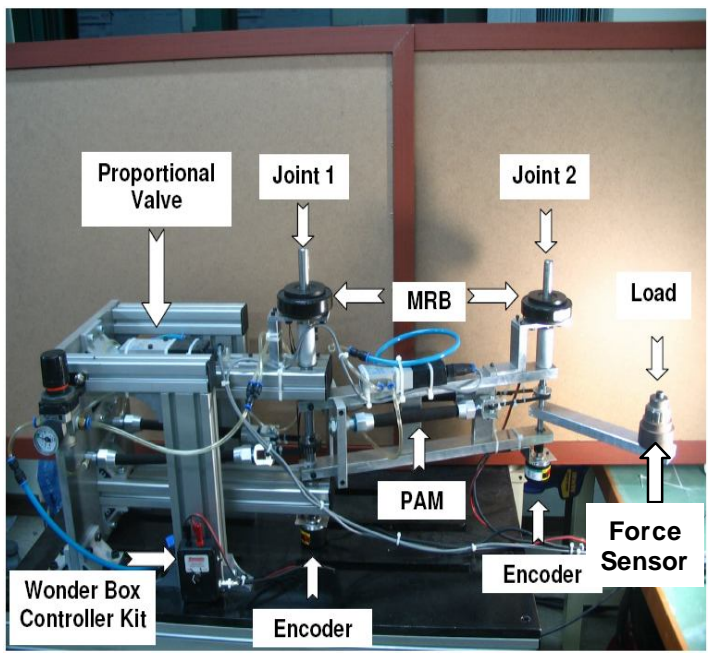

Figure 4. Photograph of the experimental 2-axes PAM robot arm.
Table 1. The lists of experimental hardware

\begin{tabular}{|c|c|c|c|}
\hline No. & Name & Model name & Company \\
\hline 1 & Proportional valuse & MPYE-5-188IF-710B & FESTO \\
\hline 2 & $\begin{array}{c}\text { Pneumatic atificial muscle } \\
(\mathrm{z} 2)\end{array}$ & MAS-10-N-220-AA-MCER & FESTO \\
\hline 3 & D/Aboard & PCI 1720 & ADVANTECH \\
\hline 4 & MD board & PCI 1711 & ADVANTECH \\
\hline 5 & Counter board & PCIOUAD-4 & $\begin{array}{c}\text { COMPJIING } \\
\text { MEASTREMENT }\end{array}$ \\
\hline 6 & Rotary encoder & $\$ 40-8-360020$ & METRONX \\
\hline 7 & Force Sensor & CBES-10 & BONGSIN \\
\hline
\end{tabular}

3. IDENTIFICATION USING DYNAMIC

INVERSE NEURAL MIMO NARX
MODEL

In general, the procedure which must be executed when attempting to identify a dynamical system consists of four basic steps (see Fig.5)

- $\quad$ STEP 1 (Getting Training Data)

- $\quad$ STEP 2 (Select Model Structure)

- $\quad$ STEP 3 (Estimate Model)

- $\quad$ STEP 4 (Validate Model:

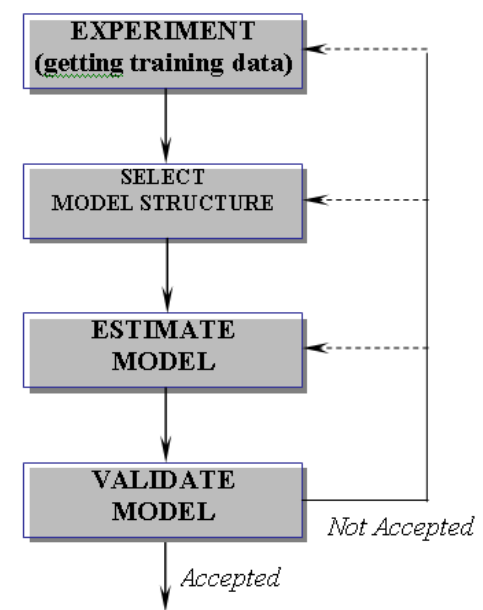

Figure 5. Neural MIMO NARX Model Identification procedure

Trang 19 
To realize Step 1, Fig.6 presents the PRBS input applied simultaneously to the 2 joints of the tested 2-axes PAM robot arm and the responding end-effector external force and joint angle outputs collected from force sensor and rotary encoders. This experimental PRBS input-output data is used for training and validating the Inverse neural MIMO NARX model of the whole dynamic two-joint structure of the 2-axes PAM robot arm as illustrated in Fig.7.

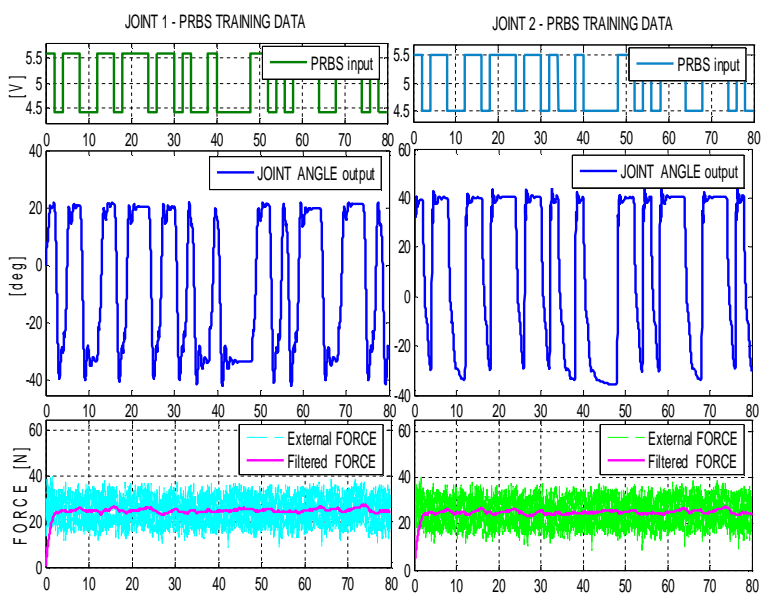

Figure 6. Input-Output training data obtained by experiment

PRBS-1(2) inputs and Force/Joint Angle outputs during (40-80)[s] will be used for training, while PRBS-1(2) inputs and Force/Joint Angle outputs in the lapse of time $(0-40)$ [s] will be used for validation purpose. The range (4.4 - 5.6) [V] and the shape of PRBS-1 voltage input applied to the $1^{\text {st }}$ joint as well as the range $(4.5-5.5)[\mathrm{V}]$ and the shape of PRBS-2 voltage input applied to rotate the $2^{\text {nd }}$ joint of the 2-axes PAM robot arm is chosen carefully from practical experience based on the hardware set-up using proportional valve to control rotating joint angle of both of PAM antagonistic pair. The experiment results of 2-axes PAM robot arm force/position control prove that experimental control voltages $u_{1}(t)$ and $u_{2}(t)$ applied to both of PAM antagonistic pairs of the 2-axes PAM robot arm is to function well in these ranges. Likewise, the chosen frequency of PRBS-1(2) signals is also chosen carefully based on the working frequency of the 2-axes PAM robot arm will be used as an elbow and wrist 2-axes PAM-based rehabilitation robot in the range of $(0.025-0.2)[\mathrm{Hz}]$.

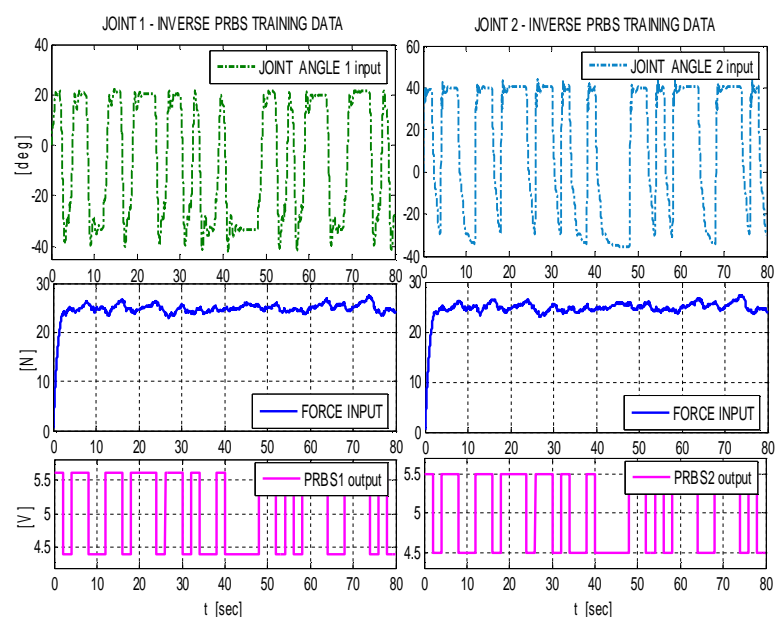

Figure 7. Inverse Neural MIMO NARX Model

Training data obtained by experiment

The $2^{\text {nd }}$ step relates to select model structure. A nonlinear neural NARX model structure is attempted. The full connected Multi-Layer Perceptron (MLPNN) network architecture composes of 3 layers with 5 neurons in hidden layer is selected (results derived from Ahn et al., 2007 [24]). The final structure of proposed Inverse neural MIMO

\section{Trang 20}


NARX11 used in proposed neural MIMO NARX FNN-PID hybrid force/position control scheme is shown in Fig.8.

The proposed neural MIMO NARX11 model structure is defined as a nonlinear neural MLPNN integrated a $1^{\text {st }}$ order ARX model (with $n_{A}=1 ; n_{B}=1$ and $n_{K}=1$ ) possessed 5 neurons in hidden layer. The activating function applied in neurons of hidden Layer and of output layer is hyperbolic tangent function and linear function respectively. Fig.9 represents the experiment block diagram for modeling and identifying the Inverse neural MIMO NARX11 model of the 2-axes PAM robot arm.

2-AXES PAM ROBOT ARM - NEURAL MIMO INVERSE NARXMODEL

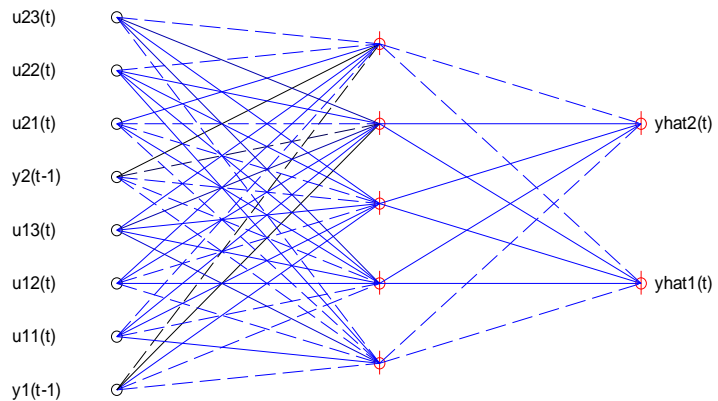

Figure 8. Structure of proposed Inverse neural MIMO NARX11 models of 2-axes PAM robot arm

In Fig.8, input values $u_{11}(t) / u_{21}(t), u_{12}(t) /$ $u_{22}(t), u_{13}(t) / u_{23}(t)$ and recurrent delayed input values $y_{1}(t-1), y_{2}(t-1)$ in neural structure of proposed neural Inverse MIMO NARX11 model will be identical to input values Joint-1 Angle $y_{1}(k)$, Joint-2 Angle $y_{2}(k)$, Force value $y_{F}(k)$ and desired recurrent delayed control voltage values $u_{1}(k-1), u_{2}(k-1)$ respectively of experimental modeling block diagram depicted in Fig.9.

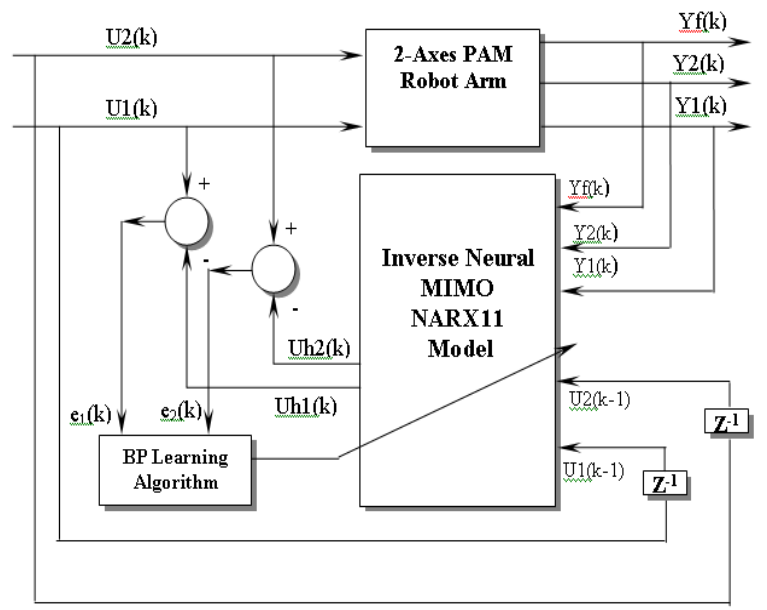

Figure 9. Block diagram for modeling of Inverse Neural MIMO NARX model of the 2-Axes PAM robot arm

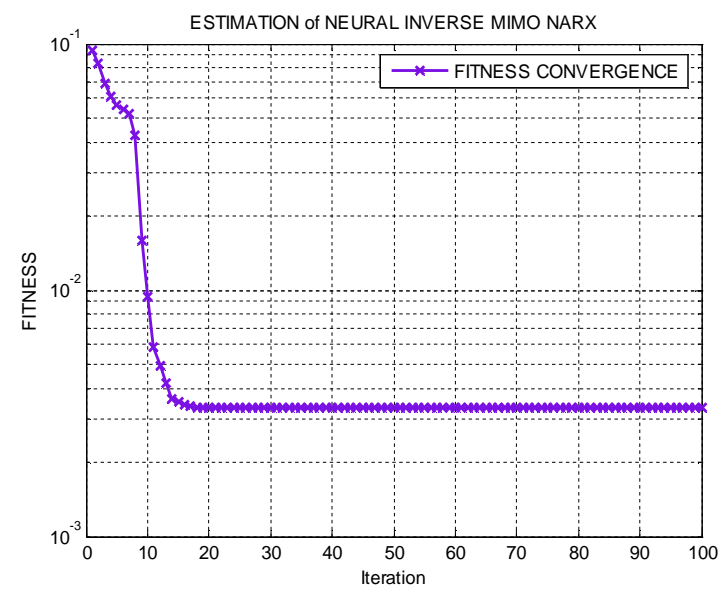

Figure 10. The fitness convergence of proposed

Neural Inverse MIMO NARX11 Model

The $3^{\text {rd }}$ step estimates trained Inverse neural MIMO NARX11 model. A good minimized convergence is shown in Fig.10 with the minimized Mean Sum of Scaled Error (MSSE) value is equal to 0.002659 after

\section{Trang 21}


number of training 100 iterations with the proposed Inverse neural MIMO NARX11. An excellent estimating result, which proves the perfect performance of resulted Inverse Neural MIMO NARX model, is also shown in Fig.11.

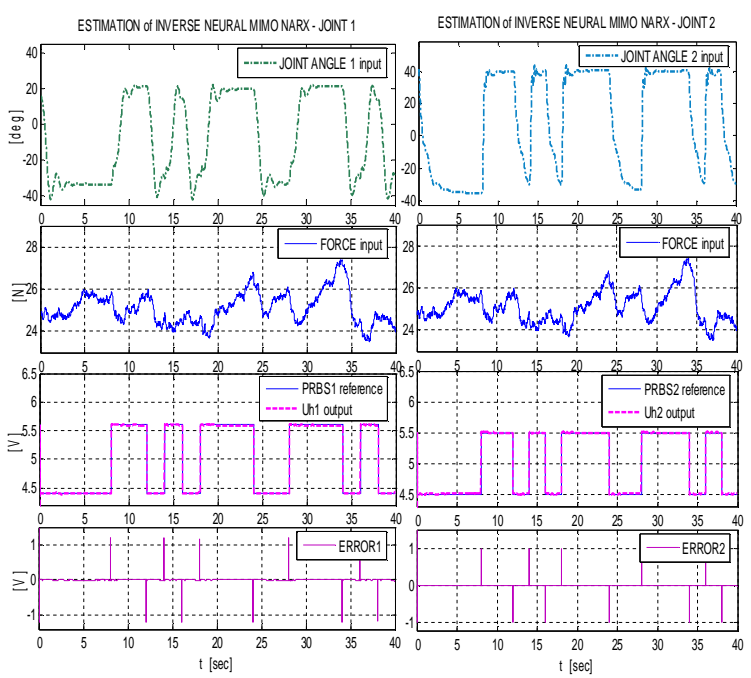

Figure 11. Estimation of 2-axes PAM robot arm

Inverse neural MIMO NARX11 Model

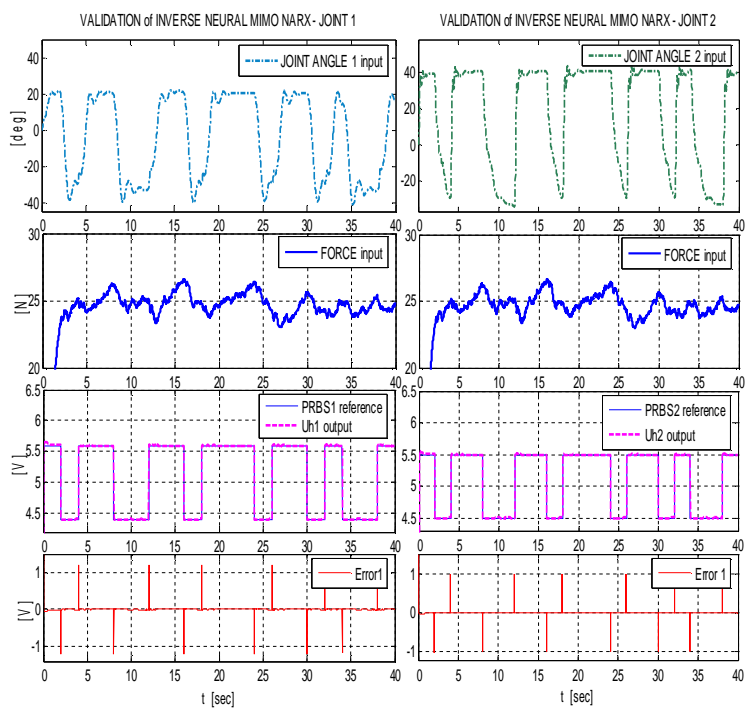

Figure 12. Validation of 2-axes PAM robot arm Inverse neural MIMO NARX11 Model
The last step relates to validate resulting nonlinear neural Inverse MIMO NARX models. Applying the same experimental diagram in Fig.6, an excellent validating result, which proves the performance of resulted Inverse Neural MIMO NARX model, is shown in Fig.12. The experimental results of the minimized errors demonstrate the good performance of the Inverse neural MIMO NARX11 Model (the excellent error < 0.01[V] for both of $U h 1 / U h 2$ control voltage values respectively applied to 2 joints of the 2-axes PAM robot arm).

Finally, Table 2 tabulates the resulting weighting values of proposed Inverse neural MIMO NARX model which can be used not only in modeling identification and simulation offline but also can be applied effectively online in model-based advanced control algorithms (Ahn and Anh, 2011)[21]. The final designed structure of proposed Inverse MIMO NARX11 model is shown in Fig.8.

\section{CONCLUSIONS}

In this study, a new approach of recurrent neural networks, proposed neural Inverse MIMO NARX model firstly utilized in modeling and identification of the highly nonlinear 2-axes pneumatic artificial muscle (PAM) system, has successfully overcome the contact force variations, coupled effect and nonlinear characteristic of the 2-axes PAM robot arm system. The 2-axes PAM robot arm's coupled dynamics was taken into account. Results of training and testing on the complex dynamic systems such as 2-axes PAM

Trang 22 
robot arm show that the newly proposed neural Inverse MIMO NARX model presented in this study is quite suitable to be applied for the modeling and identification not only the 2-axes PAM robot arm but also other nonlinear dynamic systems.

\section{Acknowledgment}

This research was partially supported by the NAFOSTED and the DCSELAB of Viet Nam.

Table 2. Resulted weights of Inverse neural MIMO NARX11 - Total Number of weighting values $=57$

\begin{tabular}{|c|c|c|c|c|c|c|c|c|c|c|c|c|c|}
\hline & \multicolumn{8}{|c|}{$\mathrm{w}_{\mathrm{ji}}$ - weights of Input Layer } & \multirow{2}{*}{$\begin{array}{c}w_{i 0}- \\
\text { weights } \\
\text { of Bias } \\
\text { Input } \\
\text { Layer } \\
0\end{array}$} & \multirow{2}{*}{\multicolumn{2}{|c|}{\begin{tabular}{c|c|} 
Whi- $_{\text {- }}$ & $\mathrm{W}_{10^{-}}$ \\
weights & weight \\
of & of Bias \\
Hidden & Hidden \\
layer & layer
\end{tabular}}} & \multirow{2}{*}{\multicolumn{2}{|c|}{$\begin{array}{c}\text { Whi - } \\
\text { weights } \\
\text { of } \\
\text { Hidden } \\
\text { layer } \\
k\end{array}$}} \\
\hline $\begin{array}{l}i \\
i\end{array}$ & 1 & 2 & 3 & 4 & 5 & 6 & 7 & 8 & & & & & \\
\hline 1 & .0 .037921 & 0.20484 & .0 .2089 & 0.55369 & 0.0 .4928 & $-0.138 \%$ & 0.25826 & -0.088107 & .0 .099618 & $\cdot 0.0397$ & & .0 .0330 & \\
\hline 2 & -0.2351 & $-0.2554 ?$ & 0.10469 & 0.029841 & .0 .069745 & 0.19078 & 0.027922 & 0.54659 & 0.098582 & 0.020958 & & 0.017458 & \\
\hline 3 & 0.21554 & 0.16316 & .0 .1149 & 0.10008 & -0.49048 & -0.14348 & 0.16629 & 0.084639 & -0.10486 & 0.06978 & & 0.05482 & \\
\hline 4 & 4.3791 & 0.4216 & $0.08270 ?$ & $1.22 \%$ & -2.0977 & $.0 .326 \%$ & 0.024291 & 1.012 & .6 .4181 & 1.0668 & & 0.88977 & \\
\hline 5 & .5 .024 & -0.14006 & 0.10937 & 0.4001 & 3.152 & 0.1362 & .0 .097036 & 0.31697 & 9.0235 & -0.6817 & & 0.56808 & \\
\hline 0 & & & & & & & & & & & 3.9315 & & $4.10 \%$ \\
\hline
\end{tabular}

\title{
NHẬN DẠNG HỆ ĐỘNG HỌC MIMO SỬ DỤNG MÔ HİNH MIMO NEURAL NARX NGƯợC
}

\author{
Hồ Phạm Huy Ánh ${ }^{(1)}$, Nguyễn Thanh Nam ${ }^{(2)}$
}

(1) Trường Đại học Bách Khoa, ĐHQG-HCM

(2) ĐHQG-HCM

TÓM TÄT: Bài báo khảo sát ứng dụng mô hình neural MIMO NARX để cải thiện chất lương nhận dạng hệ tay máy phi tuyến 2 bậc dùng bắp thịt khi nén nhân tạo (PAM). Các yếu tố nhu biến động của lục tiếp xúc hay các ảnh huởng ghép cặp phi tuyến của 2 khớp nối của tay máy sẽ được nhận dạng đầy đủ bởi mô hình neural MIMO NARX ngược thông qua bộ dũ liệu huấn luyện thực nghiệm đầu vào đầu ra. Lần đầu tiên, mô hình động học nơ rôn MIMO NARX ngược của tay máy 2 bậc dùng bắp thịt khi nén nhân tạo (PAM) được khảo sát hoàn chỉnh. Các kết quả cho thấy mô hình động học thông minh được đề xuất, được huấn luyện bằng thuật toán Lan Truyền Nguợc (BP learning algorithm) cho chất luợng tốt với độ chính xác khi nhận dạng rất cao. Mô hình động học no rôn MIMO NARX ngược cho

Trang 23 
thấy chúng có thể dùng hiệu quả trong nhận dạng không chỉ hệ tay máy PAM 2-bậc mà cho cả các hệ cơ động học phi tuyến đa biến khác.

Keywords: mô hình động học, bắp thịt khí nén nhân tạo (PAM), tay máy PAM 2 bậc, nhận dạng mô hình ngươc, mô hình nơ rôn MIMO NARX ngươc, thuật toán lan truyền ngược (BP).

\section{REFERENCES}

[1]. Noritsugu, T., Tanaka, T., Application of rubber artificial muscle manipulator as a rehabilitation robot, IEEE/ASME Trans. Mechatronics, 2, 4, 259-267 (1997).

[2]. Krebs, H. I., Hogan, N., Aisen, M. L., Volpe, B. T., Robot-aided neurorehabilitation, IEEE Trans. Rehab. Eng., 6, 1, 75-87 (1998).

[3]. Ju, M.S., Lin, C.C. K., Chen, J.R., Cheng, H.S., Lin, C.W., Performance of elbow tracking under constant torque disturbance in stroke patients and normal subjects, Clinical Biomech., 17, 640-649 (2002).

[4]. Caldwell, D. G., Tsagarakis, G. N., Development and Control of a SoftActuated Exoskeleton for Use in Physiotherapy and Training, J. of Autonomous Robots, 15, 1, 21-33 (2003).

[5]. Kobayashi, H., Uchimura, A., Shiiba, T., Development of Muscle suit for Upper Body, Proceedings Intelligent Robots and Systems (IROS 2003-IEEE Int. Conference), 4, 3624-3629 (2003).

[6]. Noritsugu, T., Sasaki, D., Takaiwa, M., Rehabilitation Robotics: Development of Active Support Splint driven by Pneumatic Soft Actuator (ASSIST), Proceedings of 2005 IEEE Int. Con. On Robotics and Automation, Barcelona, Spain, (2005).
[7]. Lilly, J. H., Adaptive tracking for pneumatic muscle actuators in bicep and tricep configurations, IEEE Trans. Neural Syst. Rehabil. Eng., , 11, 3, 333-339 (2003).

[8]. Nagaoka, T., Konishi, Y., Ishigaki, H., Nonlinear optimal predictive control of rubber artificial muscle, Proc. SPIE-Int. Soc. Opt. Eng., 2595, 54-61 (1995).

[9]. Repperger, D. W., Johnson, K. R., Phillips, C. A., VSC position tracking system involving a large scale pneumatic muscle actuator, Proc. IEEE Conf. Decision Control, Tampa, FL, $4302-4307$ (1998).

[10]. Ahn, K. K., Thanh, T. D. C., Intelligent phase plane switching control of pneumatic artificial muscle manipulators with magneto-rheological brake, Mechatronics, 16, 2, 85-95 (2006).

[11]. Carbonell, P., Jiang, Z. P., Repperger, D. W., A fuzzy backstepping controller for a pneumatic muscle actuator system, Proc. IEEE Int. Symposium Intelligent Control, Mexico City, 353-358 (2001).

[12]. Lilly, J. H. and Chang, X. Tracking control of a pneumatic muscle by an evolutionary fuzzy controller. IEEE Intell. Automat. Soft Comput., Sep.2003, vol. 9, no. 3, pp. $227-244$.

Trang 24 
[13]. Lin, C.T., Lee, C.S.G., Neural NetworkBased Fuzzy Logic Control and Decision System, IEEE Transactions on Computers, 40, 12, 1320-1336 (1991).

[14]. Karakasoglu, A., Sudharsanan, S.I., Sundareshan, M.K., Identification and decentralized adaptive control using dynamical neural networks with application to robotic manipulators, IEEE Trans. on neural networks, 4, 6, 919-930 (1993).

[15]. Katic, D.M., Vukobratovic, M. K., Highly efficient robot dynamics learning by decomposed connectionist feed-Inverse control structure, lEEE Trans. on syst. man and cybern., 25, 1, 145-158 (1995).

[16]. Lewis, F. L., Jagannathan, S., Yesildirek, A., Neural network control of robot manipulators and nonlinear systems, Taylor \& Francis (1999).

[17]. Boerlage, M., Steinbuch, M., Lambrechts, P., van de Wal, Model-Based FeedForward for Motion Systems, Proceedings of IEEE Conference on Control Applications, 1, 1158 - 1163 (2003).
[18]. Pham, D.T., Fahmy, A.A., Neuro-fuzzy Modeling and Control of Robot Manipulators for Trajectory Tracking, 16th IFAC WORLD CONGRESS, Prague, 4-8 (2005).

[19]. Ahn, K. K., Anh, H.P.H., System modeling and identification of the two-link pneumatic artificial muscle (PAM) manipulator optimized with genetic algorithm, Proceedings 2006 IEEE-ICASE Int. Conf., Busan, Korea, 4744-4749, (2006).

[20]. Ahn K.K., Anh H.P.H., A new approach of modeling and identification of the pneumatic artificial muscle (PAM) manipulator based on recurrent neural network, Proceedings IMechE, Part I: Journal of Systems and Control Engineering, 221(I8), 1101-1122 (2007).

[21]. Ahn K.K., Anh H.P.H. Compliance ForcePosition Control of the 2-Axes PAM-based Rehabilitation Robot Using Neural Networks, Journal of ISA Transactions, (2011). 